QUADERNS DE FILOSOFIA VOL. IV NÚM. I (20I7): 57-68

eISSN: 234I-3042 DOI: I0.7203/QFIA.4.I.9443

Carlos Rodríguez Gordo

Universidad de Salamanca

\title{
La educación en
}

\section{Ciberciudadanía Responsable}

\author{
(Education for Responsible Cybercitizenship)
}

Recibido: 28/12/16. Aceptado: 18/4/17

Resumen: Ante el fortalecimiento del papel de la tecnología en la sociedad actual, se hace necesario pensar el rol de aquella desde un punto de vista más radical, examinando el fenómeno mismo y sus consecuencias. La Filosofía tiene un papel fundamental a la hora de examinar y proponer alternativas educativas que contemplen las particularidades del proceso formativo del individuo y su papel como ciudadano en una sociedad profundamente mediada por la tecnología. Partiendo de las afirmaciones de algunos autores como Chomsky, Benkler, Prensky, Ortega y Gasset, Habermas y, especialmente, Hans Jonas, propongo el concepto de "Ciberciudananía Responsable" como marco crítico del Homo Tecnologicus, centrado en el pensar más que en el hacer y que pivota sobre tres temas fundamentales: alfabetización digital, fomento de la participación en red y uso de tecnologías libres.

Abstract: Given the strengthening of the role of technology in today's society, it is
necessary to think about the role of technology from a more radical point of view,
examining the phenomenon itself and its consequences. Philosophy has a funda-
mental role in examining and proposing educational alternatives that contemplate
the particularities of the individual's formative process and his role as a citizen in
a society deeply mediated by technology. Relying on the views of authors such as
Chomsky, Benkler, Prensky, Ortega y Gasset, Habermas and especially Hans Jonas,
I propose the concept of "Responsible Cybercitizenship" as a critical framework of
Homo Technologicus, which focuses on thinking rather than doing and hinges on 
three fundamental themes: digital literacy, promotion of online participation, and use of open source technologies.

Palabras clave: tecnología, educación, ciberciudadanía, ética, responsabilidad. Keywords: technology, education, cybercitizenship, ethics, responsibility.

$\mathrm{N}$

oam Chomsky (2009) se preguntaba en La (Des)educación sobre el propósito de los sistemas educativos, para concluir que existen dos modelos: uno directamente heredado de la Ilustración cuyo culmen se encuentra en la investigación, la creación y el respeto por el pasado para comprender el futuro, cuyo fin fundamental es enseñar a los individuos a aprender por sí mismos. Otro, entendiéndola como adoctrinamiento, un sistema capaz de colocar a los individuos en un marco en el que se limiten a acatar órdenes y acepten las estructuras dadas sin ningún tipo de pensamiento crítico.

Casi todas las reformas educativas estatales se han limitado a convertir uno en otro, ańadiendo más adoctrinamiento, menos formación vocacional y encareciendo los estudios de forma que, ya desde jóvenes, su endeudamiento oprima a los estudiantes y les empuje al conformismo. Tradicionalmente, ambos modelos estaban instaurados en los diferentes niveles de estudios, dejando el primero, el ilustrado, en manos de la Universidad, cuyo máximo exponente era la investigación por cuenta propia o en cooperación con otros.

La progresiva popularización de las tecnologías de la información y la comunicación ha supuesto, por un lado, una enorme crítica a las estructuras tradicionales pero, por otro, también ha significado la institucionalización de determinadas actitudes implicando la práctica fagocitación de las mismas. Algo así parecía entrever el filósofo norteamericano cuando afirmaba que los impactos de la tecnología ya no eran tan importantes como los ocurridos años atrás. Chomsky ponía el ejemplo del enorme cambio que supuso el paso del correo en barcos de vela a la invención del telégrafo. Pero no es una cuestión de impacto. Es una cuestión de comprensión. La progresiva aceleración propia de la Modernidad, promovida por la constante innovación tecnológica, ha supuesto que los hitos se acerquen en el tiempo e incluso convivan, lo que hace difícil su valoración y pueda parecer que su impacto no es tan "drástico" como en periodos anteriores. Sin embargo no es así.

Son grandes los cambios sociales que se esperan. En primer lugar, las prácticas crowd, que van a horizontalizar numerosos procesos que van desde la creación a la distribución, pasando por la financiación o la promoción. En segundo, las licencias comunales creativas (conocidas como Creative Commons), que poco a poco van creando un sistema de transmisión cultural alternativo 
al más restringido y dependiente del interés monetario copyright. Por último, pero no por ello menos importante, el abaratamiento de los dispositivos multimedia con acceso a Internet al margen del escritorio, como los lectores de tinta electrónica, las tabletas o los móviles inteligentes, que están naturalizando el consumo de material cultural e informacional y multiplicando las posibilidades de penetración e inmediatez de ambos.

No debemos obviar, no obstante, el importante factor que supone la doble brecha digital, tanto económica, que limita el acceso a la tecnología por su costo, como simbólica, que impide el desenvolvimiento fluido por parte de los no nativos en términos de Mark Prensky (200I). Llaman especialmente la atención los datos referidos al incremento de digitalización de actividades. Cada día que pasa son más y más las actividades que realizamos a través de la tecnología: comunicación, gestión bancaria, administración electrónica, consumo de material cultural, comercio electrónico... Y esta cantidad no solo es bastante alta sino que crece a un ritmo cercano al 10\%, lo que implica que, en menos de una década, multiplicaremos por dos nuestra actividad en red. Esto supone dos cosas simultáneamente: crece la importancia de la tecnología en el hacer humano y se agranda la diferencia a ambos lados de la brecha.

Aun así, sorprende que la más importante de las tareas humanas, la educación, sea uno de los ámbitos en los que la tecnología aún no ha penetrado convenientemente. Y en el caso particular de la filosofía no es diferente. No me refiero, claro está, a poner un ordenador con powerpoint en una clase presencial, ni siquiera a las formas de comunicación entre los grupos implicados en el proceso educativo (mensajería inmediata, correo electrónico, etc.). Me estoy refiriendo a integrar de forma sustancial la tecnología, tanto como herramienta, como fuente, como proceso y también como materia. Y es ahí donde la Filosofía y su pedagogía tienen un rol potencialmente esencial. Especialmente en aquello que atañe a la formación ética y cívica.

El papel fundamental de las tecnologías en la educación debería pivotar en torno al concepto de PLE, Personal Learning Enviroment, un concepto rescatado en 2001 en Reino Unido y que trata de denominar a aquellos sistemas por los cuales se ayuda a los estudiantes a tomar el control de su propia enseñanza, incluyendo soporte para configurar sus propias metas, gestionar tanto el contenido como el proceso de aprendizaje así como la comunicación con los otros (Castañeda y Adell 2013). Desde un punto de vista técnico, integra un gran número de tecnologías "2.0" que Downs describe como nudos de contenido conectados a otros servicios de creación de los mismos. El principal cambio con respecto a un entorno tradicional es que no se trata de aplicaciones institucionales, sino que son parcelas personales adaptadas a los intereses y necesidades del alumno. 
Anteriormente, el grueso de la educación constaba de unos contenidos que hoy son accesibles prácticamente de forma ubicua, y no solo eso, sino que cualquiera de las enseñanzas pueden ser completadas, recreadas y comentadas en tiempo real por cualquiera de los estudiantes. Fuentes diversas, perspectivas muy heterogéneas (y heterodoxas), con múltiples orígenes (unos mejores que otros) y formatos casi infinitos.

Toda esta época de "sobreabundancia" informativa (WelLeR 20I I, 22336) cambia para siempre la forma que tenemos de concebir la educación, permitiendo una gran capacidad de personalización que los sistemas tradicionales no contemplan ni, de hecho, soportan. Algunos autores llegan a afirmar que ambos modos de aprendizaje pueden tener incluso aspectos incompatibles lo que lleva, indefectiblemente, a que haya sociedades a las que el método tradicional ya no les sirve (Collins y Halverson 20 Io, 18-27). ¿Y por qué no sirve? Porque Chomsky (en esto) se equivocaba. Los cambios que supone la penetración de la tecnología en la sociedad son tan drásticos o más que aquellos que citaba.

Basta ver la infiltración de la tecnología en la sociedad en cualquiera de los índices harto conocidos para darse cuenta que no ha lugar para una educación que no sea substancialmente tecnológica en nuestra sociedad de hoy: política, economía, relaciones personales, consumo, etc., son demasiados aspectos tecnológicos como para darlos de lado. Y se está haciendo. Los actores tecnológicos aún no han revolucionado la educación de la misma forma y con tanta fuerza como ya lo han hecho en otros muchos aspectos de nuestra sociedad. Y va a ocurrir, o mejor dicho, ya está ocurriendo.

El problema es que la orientación política de las reformas educativas está fortaleciendo el papel de la tecnología en la educación como mera adquisición de destrezas prácticas, orientadas al desarrollo de competencias más relacionadas con la artesanía, la industria o el diseño, en definitiva, en la ciencia aplicada, que en la comprensión profunda del propio fenómeno tecnológico. Un papel que atañe a la disciplina filosófica no solo desde asignaturas relacionadas con Ciencia, Tecnología y Sociedad, sino desde otras más radicales como la Ética, la Educación Cívica, la Estética o la propia Historia de la Filosofía.

Es un problema de definición. Se ha olvidado qué significa "educar". Y no me estoy refiriendo a pedantes etimologías, sino al papel de la educación hoy. O, para no ser falaz, el que debería tener. La afirmación se comprende mejor con las palabras de Yochai Benkler, en Wealth of Networks cuando habla de la caja negra:

En un extremo, imagina una casa como una caja negra con ninguna capacidad de comunicación salvo una —la conexión de banda ancha por cable-. Cual- 
quier cosa que pase por él es, a efectos prácticos, "el estado del mundo", en lo que concierne al conocimiento de los habitantes de esa casa. En esta situación extrema, la diferencia entre un tubo completamente neutral que lleve grandes cantidades de información indiscriminadamente, y otro que esté ligeramente controlado por el operador de cable es muy grande en lo que atańe a la autonomía de los habitantes de la casa. Si la tubería es indiscriminada, entonces las elecciones de los usuarios determinan qué saben; sus decisiones en base a ese conocimiento podríamos decir que son autónomas, al menos en la medida en que ser o no autónomas es una función del estado del conocimiento del agente cuando toma una decisión. Si el tubo está controlado minuciosamente y manipulado a propósito por el operador de cable, por contraste, las decisiones que los individuos tomen en base a ese conocimiento que han adquirido gracias a ese tubo dependen sustancialmente de las elecciones del operador, no de los usuarios. En otro extremo, si cada agente tiene docenas de canales alternativos de comunicación en casa, y conoce como se maneja el flujo de información de cada uno, entonces la introducción de modificaciones en alguno de esos canales no tiene implicaciones reales en la autonomía del agente (BENKLER 2007)

La situación que expone Benkler puede leerse a la forma platónica. Imaginemos que no es un hogar sino una conciencia la que admite tan solo unas cuantas fuentes de información. Cuantas menos fuentes sean, mayor será la identificación de estas con la imago mundi que el individuo posee.

Según el "Estudio General de Medios" de 1 de abril de 2013, la Red ya era el tercer medio de comunicación con mayor penetración, un 48,6\%, solo detrás de la televisión y la radio. Además, se percibía la tendencia a sustituir horas de televisión, sobre todo, por conexiones a Internet. No en vano, en muchas franjas de edad, Internet ya era la primera elección. ${ }^{2}$ Tendencias estas que se han confirmado y acentuado: la navegación es cada vez más profunda (se visitan mayor número de sitios) y se dedica más tiempo a ello.

La mayor cantidad de tiempo de uso de tecnologías de la Información y la Comunicación se dedica a la comunicación directa y a los medios de comunicación social (o redes sociales), que se están convirtiendo en la principal fuente de información para muchos de los usuarios, teniendo en cuenta que dicha información está avalada por la procedencia y confianza entre usuarios lo que supone que la mayor parte de ese conducto del que hablaba Benkler es

${ }^{1}$ Entrega de resultados EGM 1a ola 2013. Madrid: Asociación para la Investigación de Medios de Comunicación, 22/04/2013. Disponible en http://www.aimc.es/Entrega-de-resultadosEGM-1\%C2\%AA-ola,1254.html [Consultado el 28/12/2016].

${ }^{2}$ Audiencia de Internet en el EGM (febrero-marzo 2013). Madrid: Asociación para la Investigación de Medios de Comunicación, 22/04/2 013. Disponible en http://www.aimc.es/Entregade-resultados-EGM-1\%C2\%AA-ola,1254.html [Consultado el 28/12/2016]. 
Internet. Con la lectura platónica colegimos que una gran parte de la imagen del mundo de los ciudadanos de hoy se produce a través de mecanismos tecnológicos. Además, esta cantidad es creciente debido a la continua mejora de las redes de telecomunicaciones, los dispositivos de acceso, la minimización de la brecha tecnológica... que suponen un aumento de la penetración. Con lo cual, el problema del distanciamiento entre sociedad y sistema educativo, en ciertas condiciones, no tiene visos de solución sin un cambio de naturaleza estructural.

Las tecnologías de la información, hemos visto, incluso condicionan no solo la imagen del mundo que un individuo tiene en base a la cantidad de información que recibe, como afirma Benkler en el texto citado, sino que esos datos modifican también la autoconcepción, la propia identidad, además de suponer un amplio campo de actividad laboral y educativa. En cierta forma, se recupera la idea de la "desconexión" como marginación propia del cyberpunk:

ellos se iban a encargar de que no volviese a trabajar. Le dańaron el sistema nervioso con una microtoxina rusa de los tiempos de la guerra. Atado a una cama en un hotel de Memphis, el talento se le extendió micrón a micrón y alucinó durante treinta horas. El daño fue mínimo, sutil, y totalmente efectivo. Para Case, que vivía para la inmaterial exultación del ciberespacio, fue la Caída (Gibson 1989)

A pesar de que la tendencia indique que la enseñanza tradicional reglada va a tener menor peso en la educación personal, especialmente en los niveles más altos, gran parte de las reformas del sistema educativo han ido encaminadas a mejorar la destreza en tecnologías e idiomas extranjeros (especialmente el inglés), con el fin de aminorar la posible exclusión producida por la brecha tecnológica, así como acrecentar notablemente la productividad de los alumnos en el entorno laboral. Algo beneficioso a simple vista pero que oculta un error de base, ya que se centra en el desarrollo de destrezas desde un punto de vista práctico y abandona por completo la reflexión del propio proceso, que es lo que dota de verdadera autonomía al estudiante.

La defensa de la tecnología en la educación no debe tener como único objetivo mejorar la productividad de los estudiantes como si ese fuera su fin fundamental, algo que puede deducirse de las declaraciones del otrora Ministro de Educación, Cultura y Deportes, José Ignacio Wert, impulsor de la LOMCE, o los dirigentes de las patronales. ${ }^{3}$ Incluso en la propia Ley:

${ }^{3}$ Gutiérrez, C. M. 2012, "Wert: 'Los alumnos están rindiendo por debajo de sus capacidades", Madridiario, 7/06/021012. Disponible en http://www.madridiario.es/2012/Junio/educacion/edu_educacion/218093/wert-alumnos-esfuerzan-debajo-posibilidades-renovando-espanafigar-educacion-fp-dual.html [Consultado el 28/12/2016]. 
La educación es el motor que promueve el bienestar de un país; el nivel educativo de los ciudadanos determina su capacidad de competir con éxito en el panorama internacional y de afrontar los desafíos que se planteen en el futuro. Mejorar el nivel de los ciudadanos en el ámbito educativo supone abrirles las puertas a puestos de trabajo de alta cualificación, lo que representa una apuesta por el crecimiento económico y por un futuro mejor

"Futuro mejor" directamente relacionado con los puestos de alta cualificación. Una expresión tan demagógica como errada. En lugar de promover los valores individualistas y consumistas, la educación debe encargarse de producir ciudadanos en sentido estricto. La educación de ciudadanos conscientes del proceso tecnológico, un proceso que condiciona su autopercepción, sus capacidades etc. en definitiva, su libertad, es lo que yo denomino "ciberciudadanía responsable”. ¿En qué consiste la responsabilidad ciberciudadana? El citado preámbulo del segundo borrador de la citada Ley Orgánica para la Mejora de la Calidad Educativa comenzaba así:

Los alumnos son el centro y la razón de ser de la educación. El aprendizaje en las escuelas debe ir dirigido a formar personas autónomas, críticas, con pensamiento propio. Todos los alumnos tienen un sueño, todas las personas jóvenes tienen talento. Nuestras personas y sus talentos son lo más valioso que tenemos como país. Para ello, todos y cada uno de los alumnos serán objeto de atención en una búsqueda de desarrollo del talento, que convierta a la educación en el principal instrumento de movilidad social, ayude a superar barreras económicas y sociales y genere aspiraciones y ambiciones realizables para todos

Parecen dos párrafos extraídos de diferentes fuentes. Uno que hace depender la existencia de un "mundo mejor" a individuos productores más preparados y otro que relaciona la autonomía con el pensamiento crítico. Es en este segundo contexto en el que debemos entender la ciberciudadanía responsable. Cito a Ortega:

Estos actos modifican o reforman la circunstancia o naturaleza, logrando que en ella haya lo que no hay — sea que no hay aquí y ahora cuando se necesita, sea que en absoluto no lo hay-. Pues bien; estos son los actos técnicos, específicos del hombre. El conjunto de ellos es la técnica, que podemos, desde luego, definir, como la reforma que el hombre impone a la naturaleza en vista de la satisfacción de sus necesidades. Estas, hemos visto, eran imposiciones de la naturaleza al hombre. El hombre responde imponiendo a su vez un cambio a la naturaleza. Es, pues, la técnica, la reacción enérgica contra la naturaleza o circunstancia que 
lleva a crear entre esta y el hombre una nueva naturaleza puesta sobre aquella, una sobrenaturaleza (Ortega y Gasset 20io)

La cuestión que más me interesa de la reflexión orteguiana es el matiz que pone a la tradicional concepción de la técnica como dominio natural o como una "segunda naturaleza" en el sentido aristotélico más estricto, y que Heidegger entendería como la definición instrumental y antropológica cuando dice "desde el principio, el concepto de necesidad humana abarca indiferentemente lo objetivamente necesario y lo superfluo" y "para el hombre solo es necesario lo objetivamente superfluo", donde comienza a jerarquizar los ámbitos de lo humano. Una concepción que va a aquilatar Blumenberg con el rescate de Prometeo. La "necesidad" orteguiana, la "autoafirmación" blumenbergiana, o el "desvelamiento" heideggeriano son conceptos que pivotan sobre la idea central de una evolución hacia el homo technologicus cuya manera fundamental de aproximarse a lo cotidiano es desde un punto de vista tecnológico. El ser humano es, hace y se configura tecnológicamente, con lo cual, es sustancialmente dependiente de su proceso y la comprensión del mismo implica su autocomprensión.

La ciberciudadanía responsable es la actitud crítica del hombre tecnológico, la forma de hacer en la sobrenaturaleza orteguiana. Concepto que puede entenderse con matices desde la "responsabilidad" de Jonas (I995). Dos sensaciones se encuentran en la base de esta idea: miedo y desconocimiento a los efectos futuros, a las consecuencias del progreso. ${ }^{4}$

Aunque la idea de Jonas es más bien una actualización del imperativo categórico kantiano ("obra de tal modo que los efectos de tu acción no sean destructivos para la futura posibilidad de esta vida") resulta especialmente interesante la propuesta de una responsabilidad orientada al futuro, la idea subyacente de una ética "cara al futuro", y no "en el futuro":

La expresión "ética cara al futuro" no designa una ética en el futuro —una ética futura que podríamos inventar ahora para nuestros descendientes-, sino una ética actual que se ocupa del futuro, que trata de protegerlo para nuestros descendientes de las consecuencias de nuestras acciones en el presente. Esto se ha vuelto necesario porque nuestro comportamiento actual bajo el signo de una técnica global se ha convertido en tan significativo para el futuro, y en un sentido tan perjudicial, que la responsabilidad ética impone tener en consideración en nuestras decisiones cotidianas el bien de los posteriores afectados, a los que

\footnotetext{
${ }^{4}$ Para saber más sobre este particular, sugiero consultar la tesis doctoral de ARCAs DíAz, P. 2007, Hans Jonas y el principio de responsabilidad: del optimismo científico-técnico a la prudencia responsable, Granada: Editorial de la Universidad.
} 
no les podemos preguntar su opinión. La responsabilidad se deriva para nosotros de manera inintencionada de la pura dimensión del poder que ejercemos a diario al servicio de lo inmediato, pero que dejamos repercutir sin quererlo sobre tiempos venideros lejanos (Jonas i 998, 135)

A pesar de que el concepto de Jonas está directamente relacionado con la ética ecológica y el respeto por la naturaleza, se nos vuelve completamente operativo cuando afronta la acción, proponiendo la combinación de las dos ideas que estarán en la base de nuestra noción de ciberciudadanía responsable: la precaución y la libertad. "La opción de la mera abstención a actuar nos está aquí denegada. [...] Solo se pone en duda el cómo y el cuánto; y si somos y seremos sus dueños se convierte en la cuestión más seria planteada a la responsabilidad humana" serán las palabras exactas del propio Jonas (200I, 122) en “Técnica, Libertad y Poder". Una vez que acepta que no es posible otra forma de actuar que no sea técnica, propone la cuestión del "progreso con cautela" que iría en consonancia con nuestra idea de actividad en red.

¿Por qué ciberciudadanía? Porque el concepto "ciudadanía”, entendida ésta como ciudadanía republicana, conlleva una expresión de pertenencia, un sentimiento de identidad que Juan Carlos Velasco explica así:

Ciudadanía es una categoría multidimensional que simultáneamente puede fungir como concepto legal, ideal político igualitario y referencia normativa para las lealtades colectivas. Implica en principio una relación de pertenencia con una determinada politeia (o comunidad política), una relación asegurada en términos jurídicos, pero también denota una forma de participación activa en los asuntos públicos (VeLAsCo 2006, 193)

Un concepto, el de "ciudadanía", que se ha actualizado gracias a la revitalización de la noción de "sociedad civil", poseedor de una versatilidad que hace posible su integración en numerosos contextos, y cuyo matiz republicano (SADIVAN 2003, 135-58) aleja esas tradicionales imputaciones al concepto por ser excluyente y particularista. Muy en consonancia con lo que hará Habermas ( I 998) al hacer énfasis en lo público, la participación y la deliberación, herramientas clave para entender el presente y el futuro inmediato de las tecnologías de la información y la comunicación, el social networking o las prácticas crowd (Sunstein, 2003).

Entiendo el concepto "ciberciudadanía", "ciudadanía digital" o "e-ciudadanía" como aquellos derechos y deberes de los ciudadanos en el entorno de las tecnologías de la información y la comunicación. Ribble, Bailey y Ross $(2004,9)$ determinan 9 áreas de comportamiento relacionado con el uso de la tecnología: 
Estándares de conducta, intercambio de información, educación, acceso, comercio, responsabilidad, derechos, ergonomía y riesgos. Sin embargo, el matiz de responsabilidad que yo defiendo es más restringido, y tiene que ver más con los estándares educativos norteamericanos con respecto a las tecnologías de la información y la comunicación, ${ }^{5}$ pero con una reformulación más inspirada en las prácticas habituales en social media. Entiendo la responsabilidad ciberciudadana como una cuestión fundamentalmente educativa que ha de pivotar sobre tres temas fundamentales:

a) Alfabetización digital en sentido amplio: lejos de limitarse a promover la acumulación de destrezas "productivas", incidir sobre todo en la reflexión sobre los fenómenos éticos, culturales y sociales implícitos en la tecnología. Especialmente sobre aquellos que implican renuncias conscientes a derechos fundamentales.

b) Acceso y participación en red, promoviendo la intervención en el debate social y las propuestas políticas críticas y en general, el desarrollo de actitudes conscientes encaminadas al aprendizaje permanente, la colaboración y la consecución de metas personales.

c) Fomento del uso de tecnologías y licencias libres, menos influidas por los intereses comerciales, y más respetuosas con los derechos (en sentido amplio) de los usuarios.

La gran tragedia educativa es que todo el sistema se organiza exclusivamente sobre el hacer, y no sobre el pensar. Solo un sistema ignorante de la estrecha relación que tiene la libertad humana y la tecnología, y las peligrosas desviaciones que, intrínsecamente al proceso tecnológico, la amenazan, puede obviar la cuestión tecnológica desde un punto de vista reflexivo y no meramente pragmático.

Configuración de la identidad, política, salud, relaciones personales, adquisición y transmisión de cultura, organización social, etc., son procesos ya íntimamente mediados por las tecnologías de la información y la comunicación. Si, como afirmaba la corriente contrautópica, no podemos abrazar la capacidad emancipatoria de las tecnologías, como sí lo harían los movimientos contraculturales de Bay Area en los años 60, porque somos conscientes de la perversión intrínseca del proceso tecnológico-capitalista, tampoco podemos permitirnos obligar a los alumnos a efectuar un viaje en el tiempo cuando entran en sus centros escolares, porque eso sería prepararlos para el pasado y no para el futuro, es decir, "no cabe la abstención a obrar" en palabras de Jonas. Eso es algo que se debe tener en cuenta: cuando un niño entra a formar parte del sistema educativo, lo está haciendo con la confianza de que ese enorme

\footnotetext{
${ }_{5}^{5}$ Pueden consultarse en http://www.eduteka.org/pdfdir/EstandaresNETSEstudiantes2007. pdf [Consultado el 27/12/2016].
} 
esfuerzo de enculturación le suponga una mayor preparación para su propia sociedad, no para convertirlo en testigo inútil de un particular estado de cosas presente. La educación, o tiene un gran componente predictivo o es inevitablemente conservadora y, por ende, legitimadora.

Herbert Spencer tiene una muy descriptiva cita sobre el que yo entiendo como el principal objetivo de la educación:

Téngase presente que el fin de la educación es formar un ser apto para gobernarse a si mismo, no un ser apto para ser gobernado por los demás. Si los niños hubieran de ser esclavos, deberíamos acostumbrarles a la esclavitud desde su infancia, pero, puesto que están llamados a ser hombres libres, puesto que no habrá nadie a su lado para cohibirles, es menester acostumbrarles a la propia dirección (SPENCER I917, 186)

El antropólogo británico incide en la cuestión de la gobernabilidad y la libertad como las dos partes más importantes del objetivo de la educación. Nótese también como cualquiera de estas apreciaciones no puede hacerse sin pensar en el futuro. En el futuro contexto individual de cada alumno en lugar de su pasado. La educación o es prospectiva o es Historia y no puede estar al margen de las herramientas tecnológicas, sino ser una mediadora de ellas. A nadie sorprende hoy en día que se lleve a un grupo de escolares a conducir unos karts mientras se les enseñan las normas de circulación, los semáforos, etc. Es un ejemplo de mediación en el uso de herramientas. Sin embargo no se hace así con los servicios de Internet que se usan diariamente. Y se vuelve preocupante cuando, como hemos visto, de ellas depende gran parte de la futura actividad intelectual, laboral e incluso personal de los estudiantes. Los intereses corporativos, económicos y gubernamentales afectan directa e inequívocamente a los ciudadanos y hacen necesario incluir la reflexión sobre el propio proceso tecnológico para aumentar el "autogobierno", en la terminología de Spencer, la "autonomía", en definitiva, la responsabilidad ciberciudadana. Y es en esa reflexión donde reside, sin ningún género de dudas, el papel crucial de la Filosofía. 


\section{BIBLIOGRAFÍA}

ArCas Díaz, P. 2007, Hans Jonas y el principio de responsabilidad: del optimismo cientifico-técnico a la prudencia responsable, Granada: Editorial de la Universidad.

Benkler, Y. 2007, The Wealth of Networks: How social production transforms markets and freedom, New Haven: Yale University Press.

Castañeda, L. y Adell, J. 2013, Entornos personales de aprendizaje: claves para el ecosistema educativo en Red, Alcoy: Marfil.

Chomsky, N. 2009, La (Des)Educación, Barcelona: Crítica.

Collins, A. y Halverson, R. 20io, "The second educational revolution: Rethinking education in the age of technology", Journal of Computer Assisted Learning, 26 (1): 18-27.

Gibson W. 1989, Neuromante, Barcelona: Minotauro.

Habermas, J. I998, Facticidad y Validez, Madrid: Trotta.

Jonas, H. I998, "La fundamentación ontológica de una ética cara al futuro", en Pensar sobre Dios y otros ensayos, Barcelona: Herder, 135.

Jonas, H. 200 I, “Técnica, Libertad y Poder", en Más cerca del perverso fin y otros diálogos y ensayos, Madrid: Catarata, 122.

Jonas, H. 1995, El principio de responsabilidad: ensayo de una ética para la civilización tecnológica, Barcelona: Herder.

Ortega y Gasset, J. 20 io, La meditación de la técnica y otros ensayos sobre ciencia y filosofía, Madrid: Alianza.

Prensky, M. 200I, "Digital natives, digital immigrants", On The Horizon 9, Wagon: MCB University Press.

Ribble, M. S.; Bailey, G. D. y Ross, T. W. 2004, "Digital Citizenship, Adressing appropriate technology behaviour", Learning \& Leading with technology, 32: 9.

SAdivan, P. 2003, "La crítica republicana del liberalismo", en Conill, J. y Crocker,

D. A., Republicanismo y educación cívica. ¿Más allá del liberalismo?, Granada: Comares, 135-58.

Spencer, H. I917, La educación intelectual, moral y física, Valencia: F. Sempere y Compañía, 186.

Sunstein, C. S. 2003, República.com. Internet, democracia y libertad, Barcelona: Paidós.

Velasco, J. C. 2006, "La noción republicana de ciudadanía y la diversidad cultural”, Isegoría, 33: 193.

Weller, M. 200I, "A pedagogy of abundance", Revista Española de Pedagogía, 249: 223-36. 